\title{
Local segregation versus irradiation effects in high-entropy alloys : Steady-state conditions in a driven system
}

\section{Koch, Leonie}

2017-09-14

Koch , L , Granberg , F , Brink , T , Utt , D , Albe , K , Djurabekova , F \& Nordlund , K 2017 , ' Local segregation versus irradiation effects in high-entropy alloys : Steady-state conditions in a driven system ' , Journal of Applied Physics , vol. 122 , no. 10 , 105106 . https://doi.org/10.1063/1.4990950

http://hdl.handle.net/10138/297920

https://doi.org/10.1063/1.4990950

unspecified

publishedVersion

Downloaded from Helda, University of Helsinki institutional repository.

This is an electronic reprint of the original article.

This reprint may differ from the original in pagination and typographic detail.

Please cite the original version. 


\section{Local segregation versus irradiation effects in high-entropy alloys: Steady-state conditions in a driven system}

Leonie Koch, Fredric Granberg, Tobias Brink, Daniel Utt, Karsten Albe, Flyura Djurabekova, and Kai Nordlund

Citation: Journal of Applied Physics 122, 105106 (2017); doi: 10.1063/1.4990950

View online: https://doi.org/10.1063/1.4990950

View Table of Contents: http://aip.scitation.org/toc/jap/122/10

Published by the American Institute of Physics

\section{Articles you may be interested in}

Effect of valence electron concentration on stability of fcc or bcc phase in high entropy alloys Journal of Applied Physics 109, 103505 (2011); 10.1063/1.3587228

Pressure-induced fcc to hcp phase transition in Ni-based high entropy solid solution alloys Applied Physics Letters 110, 011902 (2017); 10.1063/1.4973627

Predicting solid solubility in CoCrFeNiM $(\mathrm{M}=4 \mathrm{~d}$ transition metal) high-entropy alloys Journal of Applied Physics 121, 194903 (2017); 10.1063/1.4983762

Features of primary damage by high energy displacement cascades in concentrated Ni-based alloys Journal of Applied Physics 119, 085901 (2016); 10.1063/1.4942533

Atomic displacement in the CrMnFeCoNi high-entropy alloy - A scaling factor to predict solid solution strengthening

AIP Advances 6, 125008 (2016); 10.1063/1.4971371

Interactions between coherent twin boundaries and phase transition of iron under dynamic loading and unloading

Journal of Applied Physics 122, 105107 (2017); 10.1063/1.4997320

\section{AIP| loumalof}

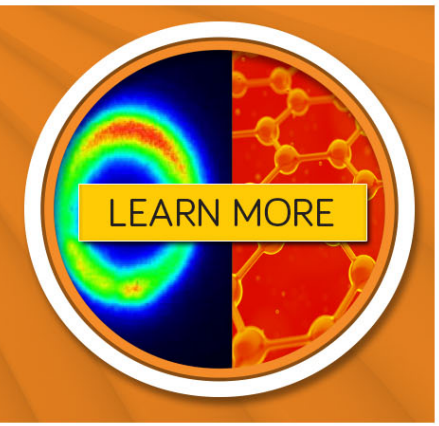




\title{
Local segregation versus irradiation effects in high-entropy alloys: Steady-state conditions in a driven system
}

\author{
Leonie Koch, ${ }^{1, \text { a) }}$ Fredric Granberg, ${ }^{2}$ Tobias Brink, ${ }^{1}$ Daniel Utt, ${ }^{1}$ Karsten Albe, ${ }^{1}$ \\ Flyura Djurabekova, ${ }^{2,3}$ and Kai Nordlund ${ }^{2}$ \\ ${ }^{1}$ Fachgebiet Materialmodellierung, Institut für Materialwissenschaft, TU Darmstadt, \\ Jovanka-Bontschits-Straße 2, D-64287 Darmstadt, Germany \\ ${ }^{2}$ Department of Physics, University of Helsinki, P.O. Box 43, Helsinki FIN-00014, Finland \\ ${ }^{3}$ Helsinki Institute of Physics, University of Helsinki, P.O. Box 43, Helsinki FIN-00014, Finland
}

(Received 19 June 2017; accepted 24 August 2017; published online 11 September 2017)

\begin{abstract}
We study order transitions and defect formation in a model high-entropy alloy (CuNiCoFe) under ion irradiation by means of molecular dynamics simulations. Using a hybrid Monte-Carlo/ molecular dynamics scheme, a model alloy is generated which is thermodynamically stabilized by configurational entropy at elevated temperatures, but partly decomposes at lower temperatures by copper precipitation. Both the high-entropy and the multiphase sample are then subjected to simulated particle irradiation. The damage accumulation is analyzed and compared to an elemental Ni reference system. The results reveal that the high-entropy alloy-independent of the initial configuration-installs a certain fraction of short-range order even under particle irradiation. Moreover, the results provide evidence that defect accumulation is reduced in the highentropy alloy. This is because the reduced mobility of point defects leads to a steady state of defect creation and annihilation. The lattice defects generated by irradiation are shown to act as sinks for Cu segregation. Published by AIP Publishing. [http://dx.doi.org/10.1063/1.4990950]
\end{abstract}

\section{INTRODUCTION}

High-entropy alloys (HEAs) constitute a relatively new class of materials, which have recently attracted considerable attention in the field of high-performance materials. ${ }^{1}$ They consist of at least four to five principal elements occurring in an equimolar or near equimolar ratio, such that their fractions do not drop below $5 \%$ or exceed $35 \% .^{2-4}$ HEAs owe their name mainly to the large contribution of configurational entropy to the Gibbs free energy. It is supposed that the influence of the entropy stabilizes random solid solutions even at lower temperatures. ${ }^{5,6}$ However, mixing enthalpies and the atomic size mismatches between the components decisively contribute to the phase selection criterion at lower temperatures ${ }^{4}$ and the formation of secondary phases can often not be completely avoided. ${ }^{7}$

The interest in high-entropy alloys is mostly triggered by their outstanding mechanical properties, making them alternatives for certain superalloys or metallic glasses. ${ }^{2,4,8-16}$ However, high-entropy alloys are not only characterized by a large configurational entropy, but also by high atomic-level stresses arising from local lattice distortions due to atomic size differences. ${ }^{17}$ It has been reported that structural and chemical disorder affects both defect kinetics and heat dissipation, which are of particular interest in the context of radiation resistant materials. ${ }^{18}$ Recent molecular dynamics (MD) simulations revealed that defect concentrations can be significantly reduced during irradiation in equiatomic multicomponent alloys as compared to elemental metals. ${ }^{19,20}$ This advantageous robustness against radiation damage can be ascribed to a reduced defect mobility and consequently a

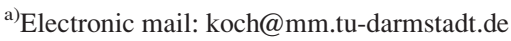

smaller growth rate of defect clusters. ${ }^{19-21}$ Additionally, chemical disorder decreases thermal conductivity, which is also assumed to prevent the formation of large defect clusters by facilitating defect annihilation. ${ }^{17,18,22}$ If, on the other hand, a HEA has a tendency towards multiphase formation at lower temperatures, particle irradiation can also drive the system into thermodynamic equilibrium and lead to precipitiation. $^{21}$ Thus, in irradiated high-entropy alloys there is a delicate interplay of thermodynamic driving forces due to configurational entropy, mixing/demixing tendencies, and irradiation-induced far-from-equilibrium conditions.

The objective of the present study is to address this issue in a model four-component $\mathrm{CuNiCoFe}$ alloy in thermodynamic equilibrium and under particle irradiation, as well as to study the transition from a single phase HEA to a multiphase or so-called compositionally complex alloy. Using a hybrid simulation scheme consisting of alternating MonteCarlo (MC) and MD steps, we start by generating a model alloy which is truly stabilized by configurational entropy at elevated temperatures. We then show that this structure decomposes at lower temperatures by precipitation of small copper clusters. Both the high-entropy and the partly decomposed samples are then subjected to a series of 1500 recoil events and the damage accumulation is analyzed and compared to elemental $\mathrm{Ni}$ as the reference system. Moreover, we show that segregation effects occur at irradiation-induced defects, promoting the formation of precipitates.

\section{METHODS}

\section{A. Interatomic potential}

We chose a CuNiCoFe alloy as a model system for HEAs with a tendency for $\mathrm{Cu}$ segregation at low temperatures. The 
embedded atom method ${ }^{23}$ (EAM) was used to describe interatomic interactions in the multicomponent system. Parametrizations for the elemental interactions were taken from Zhou et al..$^{24}$ The missing cross terms were created using the potential generator from the same authors, which is based on a single element mixture procedure. ${ }^{24}$ Because of the ferromagnetic elements, magnetism and magnetic transitions could influence the evolution of chemical ordering. EAM potentials cannot explicitly capture these effects and include the magnetic energy contributions only implicitly. For this reason, our system can only serve as a model for nonmagnetic HEAs or HEAs without magnetic transitions. In order to nonetheless ensure the reliability of the potential, we validated it against reference data in the Appendix.

\section{B. Equilibrium simulations}

Initially, atoms were distributed randomly on a regular FCC lattice of about $N=100000$ sites, such that an equimolar configuration was obtained. We applied 3-dimensional periodic boundary conditions and the initial lattice constant was approximated according to Vegard's law. ${ }^{25}$ After the construction of the initial structure, we minimized its free energy with a mixed MC/MD procedure, carried out using the open source code LAMMPS. ${ }^{26}$ For the MC steps, we used the variance-constrained semi-grand-canonical (VC-SGC) ensemble. ${ }^{27}$ This ensemble is based on the semi-grandcanonical ensemble with an added constraint on the overall composition. Thus, it resembles a canonical ensemble, but allows Gaussian variations of the concentration, the extent of which is controlled by the parameter $\kappa$. We chose a value of $\kappa=10^{3}$ in our simulations. The VC-SGC ensemble allows simulations inside miscibility gaps and has a performance advantage over the canonical ensemble, which cannot be parallelized easily. ${ }^{27}$ The miscibility is controlled via the chemical potential differences. The chemical potential differences with regard to copper were determined as $\Delta \mu_{\mathrm{Cu}-\mathrm{Ni}}$ $=0.9 \mathrm{eV}, \Delta \mu_{\mathrm{Cu}-\mathrm{Co}}=0.85 \mathrm{eV}$, and $\Delta \mu_{\mathrm{Cu}-\mathrm{Fe}}=0.7 \mathrm{eV}$ as described in Ref. 28. The values at hand lead to a miscible $\mathrm{CuNiCoFe}$ system at $800 \mathrm{~K}$.

The MC/MD simulations were performed at different target temperatures $T=800 \mathrm{~K}, 750 \mathrm{~K}, \ldots, 400 \mathrm{~K}$, with $N / 4$ MC trial moves followed by $20 \mathrm{MD}$ steps. For the MD steps, we used a timestep of $1 \mathrm{fs}$, as well as a Nosé-Hover thermostat and a Parinello-Rahman barostat at temperature $T$ and ambient pressure. In total, the simulations were run for $1000000 \mathrm{MD}$ steps (including $50000 \mathrm{MC}$ cycles), after which the potential energy was definitely converged.

\section{Irradiation simulations}

We simulated irradiation of the structures using the approach introduced in Ref. 19 with the PARCAS MD code. ${ }^{29,45,75,76}$ Simulations were started from either pure Ni, a $\mathrm{CuNiCoFe}$ cell with random element distribution, or the equilibrated sample from the MC/MD run at $400 \mathrm{~K}$. After equilibration at room temperature to zero pressure, the simulation cell size was fixed and a series of subsequent $5 \mathrm{keV}$ recoils was initiated in the cell. The recoils were performed by assigning a velocity vector with a magnitude corresponding to the recoil energy and a random direction to the atom closest to the center of the simulation cell. Each recoil was simulated for $30 \mathrm{ps}$, which was sufficient to cool the cell back down to ambient temperature by Berendsen temperature scaling ${ }^{30}$ in a thin layer at the simulation box boundaries. After this, all atom coordinates were shifted by a displacement vector with components randomly selected in the interval $\left[0, L_{d}\right]$, where $L_{d}$ is the cell size in each of the 3 dimensions $d$. After the shift, atoms outside the boundaries were wrapped back into the cell according to the periodic boundary conditions. This procedure ensures homogeneous irradiation of the entire simulation cell. Experimentally, this is comparable, e.g., to prolonged neutron or high-energy ion irradiation of a segment inside the material.

The irradiation simulations used the same interatomic potential as the MC/MD simulations, except that for small interatomic separations (well below the equilibrium nearestneighbor distance) the potentials are smoothly joined to the universal repulsive Ziegler-Biersack-Littmark (ZBL) interatomic potential. ${ }^{31}$ The ZBL-96 electronic stopping ${ }^{32}$ was applied on all atoms with kinetic energies higher than $1 \mathrm{eV}$. Monitoring of the simulations showed that about $20 \%$ of the initial recoil energy was lost to electronic stopping, i.e., the nuclear deposited damage energy per recoil is about $4 \mathrm{keV}$. The total deposited nuclear energy was extracted by summing up the damage energies and then used to calculate the displacement-per-atom (dpa) value, a standard unit for radiation damage exposure. ${ }^{33,34}$ This irradiation simulation approach has previously been demonstrated to lead to a good agreement with experiments on other HEAs. ${ }^{19,35}$

\section{Analysis of the short-range order and lattice defects}

In order to quantify the chemical ordering, we used the Warren-Cowley short-range order (SRO) parameters $\alpha_{1}$ and $\alpha_{2}$ adapted to a multicomponent alloy ${ }^{36}$

$$
\alpha_{n}^{i j}=1-\frac{P_{n}^{i j}}{c_{j}}
$$

where the subscript $n \in\{1,2, \ldots\}$ refers to the $n$th coordination sphere, $P_{n}^{i j}$ describes the conditional probability of an atom of type $j$ being adjacent to an atom of type $i$, and $c_{j}$ is the concentration of atom type $j$. A Warren-Cowley parameter of zero represents the case of an ideal solid solution with no tendency for clustering. Ordered structures (attractive SRO) can be assumed if $\alpha$ is positive, while negative values reflect preferences for segregation (repulsive SRO). We calculated the SRO parameters for the equilibrium structures as a time average over 20 snapshots taken during 2000 simulation steps. Those snapshots were minimized prior to analysis to remove thermal fluctuations and to obtain the $0 \mathrm{~K}$ groundstate values. For the irradiated cells, the SRO analysis was performed in the same manner, except that averaging was only performed where noted.

We used the open source application oviтo ${ }^{37}$ for the analysis and visualization of simulation results: Atomic volumes were calculated by Voronoi tesselation of minimized samples, ${ }^{38-41}$ defective atoms were marked by common neighbor analysis (CNA) with an adaptive cutoff, ${ }^{42,43}$ and dislocation 
lines were identified using the dislocation extraction algorithm (DXA). ${ }^{44}$ Additionally, we also performed a Wigner-Seitz cell defect analysis. ${ }^{45}$ This analysis constructs the space-filling Wigner-Seitz cells of the perfect underlying FCC lattice, labeling empty cells as vacancies and doubly filled ones as interstitials. Since this analysis is space-filling, it gives a definite determination of whether a defect region is of vacancy or interstitial type (complex defects may involve both, but then the difference in the number of vacancies vs. interstitials of the same cluster determines its character ${ }^{46}$ ) The supplementary material provided by this analysis helps to identify point defects, which cannot be treated by DXA.

\section{RESULTS AND DISCUSSION}

\section{A. CuNiCoFe in thermodynamic equilibrium}

Figure 1 shows the final structures after equilibration in the VC-SGC ensemble at $800 \mathrm{~K}$ (left) and at $400 \mathrm{~K}$ (right). The configurations differ slightly in their atomic arrangement. Whereas $\mathrm{Cu}$ (red atom type) appears to be randomly distributed at $800 \mathrm{~K}$, it tends to form small clusters in the structure at $400 \mathrm{~K}$, indicated by the white arrow. The Warren-Cowley parameters at both temperatures are shown in Fig. 2. All $\alpha_{n}$ values more or less deviate from the ideal solid solution in either the positive or negative direction. This behavior is most pronounced for $\mathrm{Cu}$. On average, we find a Warren-Cowley parameter for $\mathrm{Cu}-\mathrm{Cu}$ of -0.15 for the first neighbor shell at $800 \mathrm{~K}$, indicating a slight tendency to form small $\mathrm{Cu}$ clusters (positive $\Delta H_{\text {mix }}$ ). Accordingly, all other $\alpha_{1}^{\mathrm{Cu}-j}$ values are positive. Except for Fe-Fe interactions, all remaining parameters are slightly negative, indicating miscibility of $\mathrm{Ni}, \mathrm{Co}$, and $\mathrm{Fe}$. The $\alpha_{2}$ parameter exhibits no strong indication for medium-range ordering at $800 \mathrm{~K}$.

If we repeat the analysis in the same sample equilibrated at $400 \mathrm{~K}$, we see that the ordering tendencies become more distinct and extend into the second neighbor shell. Although the Warren-Cowley parameters for non-Cu pairs also change in magnitude, they still indicate an approximately random solid solution of $\mathrm{Co}, \mathrm{Ni}$, and $\mathrm{Fe}$ without any remarkable clustering. This can also be inferred from the snapshots in Fig. 1. Figure 2(c) further shows that the $\mathrm{Cu}$ segregation tendencies increase non-linearly with decreasing temperature. Note that we do not observe any structural phase transitions, such as a transition from an FCC to a BCC lattice or even amorphization.

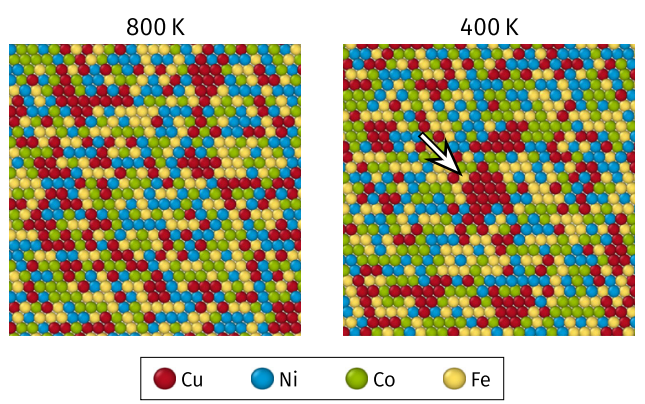

FIG. 1. Snapshots of the CuNiCoFe alloy, equilibrated in the VC-SGC ensemble at $800 \mathrm{~K}$ (left) and $400 \mathrm{~K}$ (right). The arrow highlights a clustering of copper atoms, indicating that phase separation occurs at lower temperatures.

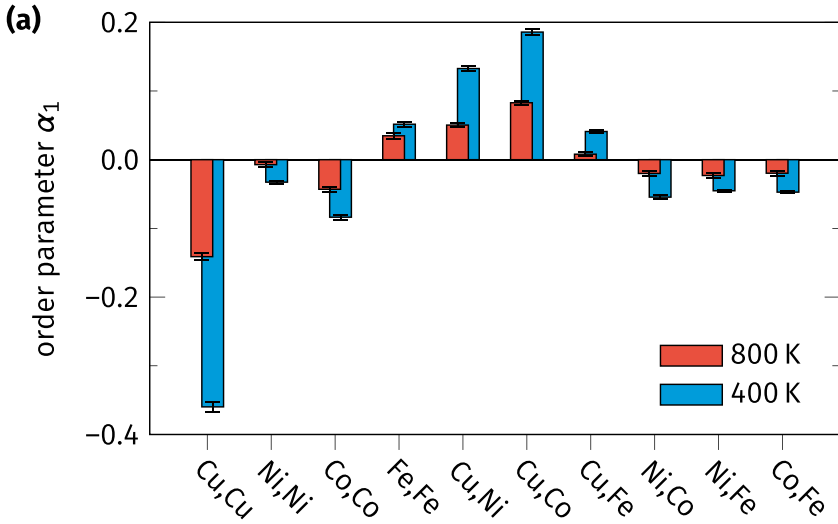

(b)
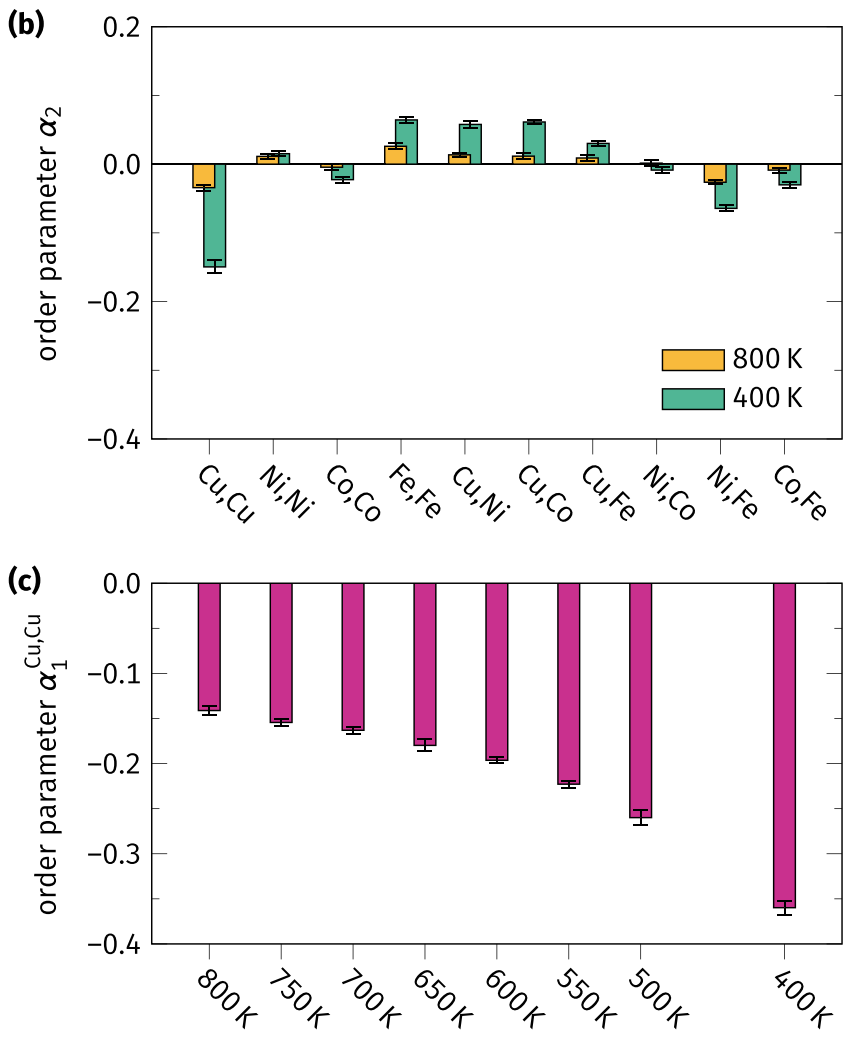

FIG. 2. Warren-Cowley parameters $\alpha_{1}$ (a) and $\alpha_{2}$ (b) for the CuNiCoFe alloy system at 800 and $400 \mathrm{~K}$. (c) The change of $\alpha_{1}^{\mathrm{Cu}, \mathrm{Cu}}$ with temperature. Increasing $\mathrm{Cu}-\mathrm{Cu}$ ordering tendencies can be observed with decreasing temperature. All values are averaged over 20 snapshots at $0 \mathrm{~K}$, with the error bars representing the standard deviation.

These results may be interpreted as follows: The contribution of the configurational entropy at $400 \mathrm{~K}$ is insufficient to compete against the enthalpy of mixing. Since the composition of the system is fixed, it will demix by locally forming copper-rich clusters. The formation of two separate solid solution phases (Cu-rich and $\mathrm{Cu}$-depleted) has already been confirmed experimentally by Otto et $a l^{3}$ The observed decomposition is more pronounced than in the present simulation, which may either be due to the different composition in the experiment $(\mathrm{CoCrFeMnCu})$, or the higher solubility of $\mathrm{Cu}$ in the EAM potential (see Appendix). The authors found that the $\mathrm{Cu}$ segregation is driven by positive binary mixing enthalpies between copper and the remaining components. ${ }^{3}$ Thus, when decreasing the temperature in the experiment, there is a competition between the increasing thermodynamic driving 
force for decomposition and the decreasing diffusivity within the system. For this reason, HEAs often represent kinetically trapped metastable configurations at lower temperatures and local segregation occurs as an ageing phenomenon.

When comparing the atomic volumes as a function of the atom type (see Fig. 3), it can be seen that $\mathrm{Cu}$ has slightly larger values than $\mathrm{Co}, \mathrm{Ni}$, or $\mathrm{Fe}$. Both $\mathrm{Ni}$ and $\mathrm{Co}$ possess higher atomic volumes in the multicomponent system than in their elemental state. In contrast, $\mathrm{Cu}$ and $\mathrm{Fe}$ exhibit smaller atomic volumes compared to their pure structures. At $400 \mathrm{~K}$, the system starts demixing into a ternary $\mathrm{Co}, \mathrm{Ni}$, and Fe solid solution and into a copper-rich phase. The copper atoms expand slightly, while the ternary solid solution becomes slightly denser. This brings $\mathrm{Cu}, \mathrm{Co}$, and $\mathrm{Ni}$ closer to their preferred atomic volume. Only $\mathrm{Fe}$ deviates from this trend, but exhibits a positive Warren-Cowley parameter, indicating a preference for unlike neighbors. This changed chemical environment may influence the equilibrium volume.

In summary, these observations suggest that the small scale decomposition is driven largely by the mixing enthalpy at low temperatures, but additionally enhanced by atomic size effects.

\section{B. Irradiation}

Thus far, we studied the thermodynamic stability of a $\mathrm{CuNiCoFe}$ solid solution. In Sec. III A, we demonstrated by means of hybrid $\mathrm{MC} / \mathrm{MD}$ simulations that $\mathrm{CuNiCoFe}$ exhibits a tendency for decomposition at lower temperatures ( $400 \mathrm{~K})$. These results suggest that in this case a random distribution is only stabilized by kinetic inhibition. In the following, we will therefore discuss the effect of cascade events on the evolution of the chemical order, focusing on the question, if the energy that is released during recoil events enables the decomposition of $\mathrm{Cu}$. We irradiate both the equilibrated, partially segregated sample obtained by MC/MD simulation at $400 \mathrm{~K}$ ("initially

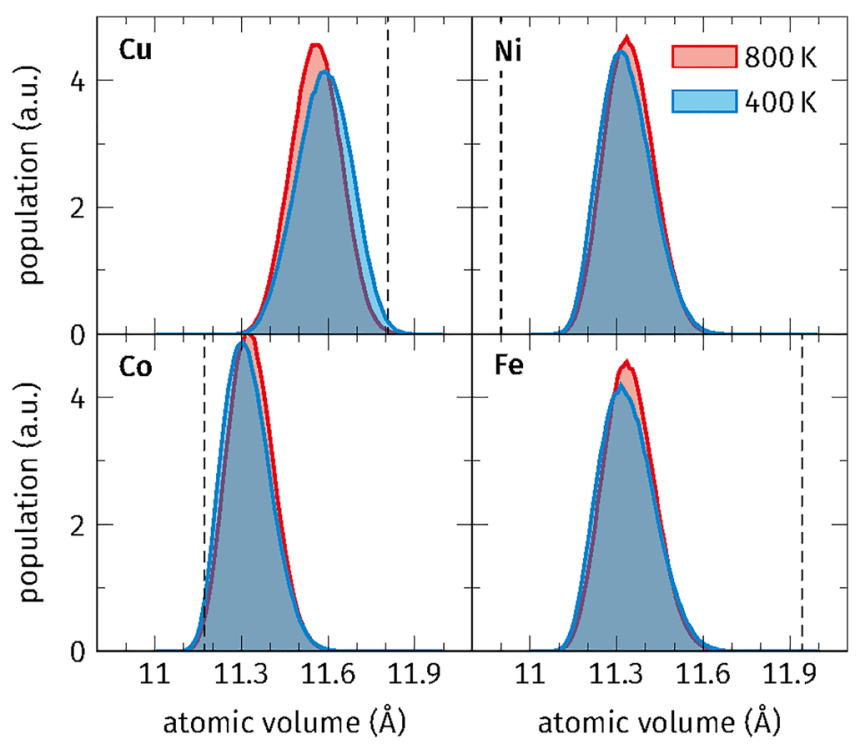

FIG. 3. Histograms of the atomic volumes at $0 \mathrm{~K}$ for the HEA equilibrated at $400 \mathrm{~K}$ (blue) and $800 \mathrm{~K}$ (red). The dashed lines indicate the ground state atomic volume of the single-element FCC structures. Atomic volumes are obtained from Voronoi tesselation ${ }^{38-41}$ of 20 snapshots at $0 \mathrm{~K}$. segregated"), and a sample with completely random element distribution ("initially random"). Additionally, we investigate the structural damage due to irradiation by comparison with a Ni sample.

\section{Lattice defects}

Earlier work comparing irradiation damage in elemental metals and disordered alloys suggests that the FCC structure is retained throughout irradiation with 1500 recoils. ${ }^{19}$ Figure 4(a) shows that this is also the case for the initially segregated HEA sample (equilibrated with MC/MD at $400 \mathrm{~K}$ ). Since the structural damage in the initially random HEA is similar, we omit this sample from the discussion at hand. For comparison, Figure 4(b) shows a pure Ni reference sample which underwent the same irradiation treatment. Most irradiation damage in these samples manifests itself by the formation of dislocation loops, stacking-fault tetrahedra, and single vacancies or vacancy clusters. Vacancy clusters and extended defect agglomerates cannot be identified by the DXA and are indicated by red defect meshes. A visualization of atom positions in Fig. 4(c) reveals that these regions are dominantly characterized by vacant lattice sites. The nature of these regions has also been confirmed by a Wigner-Seitz cell defect analysis, which shows that the positions of the distorted regions generally coincide with vacancy-type defect clusters (see videos cascade-CuNiCoFe.avi and cascade-Ni.avi in the supplementary material). These vacancy-type defects are homogeneously distributed within the structure.

The irradiation process and therefore the formation and evolution of defects can be divided into three stages. Figures 4(a) and 4(b) visualize one representative configuration for each of the three stages. Figure 4(d) depicts the defect concentration as a function of the irradiation dose. Initially, the HEA shows a higher defect accumulation than the pure Ni system, which could be caused by higher atomic level stresses within the multicomponent system. This results in a reduced defect formation energy and thus increased number of defects. ${ }^{17} \mathrm{In}$ turn, pure Ni exhibits a higher recombination rate due to an increased point defect mobility, ${ }^{18,22}$ which means that fewer defects are created per recoil event. During the first cascades, mainly point defects occur in both structures, while the concentration of dislocation-network-like structures is still small.

In the second stage, with an increase of irradiation damage, the defect concentration in pure $\mathrm{Ni}$ increases and reaches the same magnitude as in the high-entropy structure. It can be seen that apart from vacancies and stacking-fault tetrahedra, a rather large and stable dislocation loop is formed by the agglomeration of small defects during successive cascades. In general, the rate of defect annihilation depends on both the size and the spatial distribution of the defects. Small defects can be annihilated if they are hit by a single recoil event, while larger defect structures are not engulfed completely and are therefore more stable. Furthermore, the agglomeration of defects in $\mathrm{Ni}$ leads to their localization, while the small defects in the HEA are more homogeneously distributed. This means that the probability of a cascade randomly hitting a defect is higher in the HEA and a steady state of annihilation and creation is reached (see video cascade-CuNiCoFe.avi in 

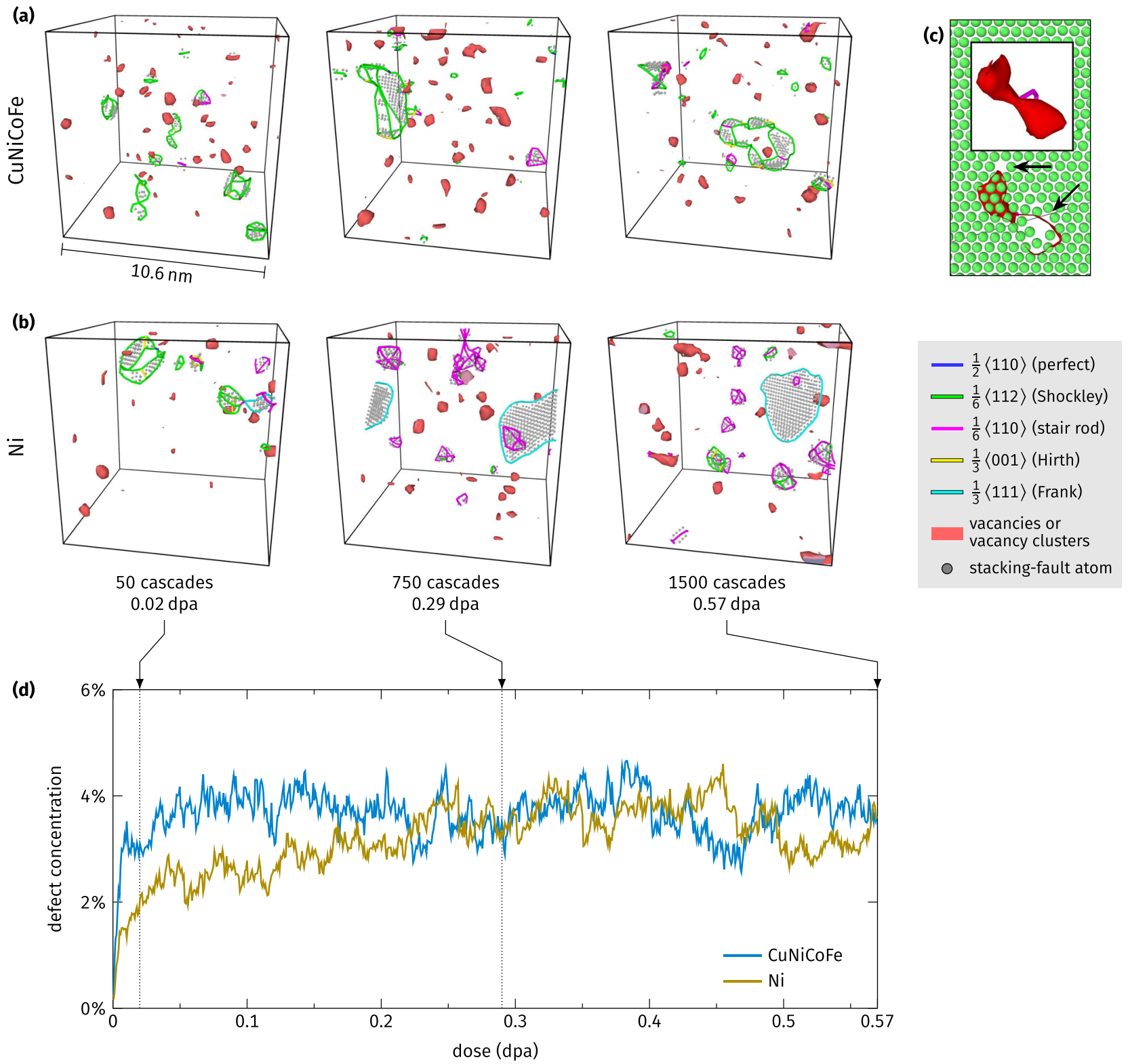

FIG. 4. Analysis of the build-up of lattice defects during irradiation. (a) DXA analysis of the initially segregated HEA at different irradiation doses. (b) The same for a Ni sample. Empty space represents the perfect FCC lattice; the structures do not collapse during irradiation. Green lines indicate $\langle 112\rangle$ partial dislocations, turquoise lines indicate a Frank loop, purple lines belong to stacking fault tetrahedra, and red surfaces enclose defects that cannot be recognized by DXA. Videos of these simulations can be found in the supplementary material (CuNiCoFe-ordered-DXA-during-irradiation.avi and Ni-DXA-during-irradiation.avi). (c) A closer look at those red regions reveals that they represent vacancies and vacancy clusters. In (d), a plot of the concentration of defective atoms as identified by CNA is shown as a function of the irradiation dose. In agreement with the DXA results, we can see that the HEA quickly reaches a high defect concentration that saturates around $4 \%$. These defects consist mostly of vacancies and small dislocation networks. Pure Ni builds up the defect concentration more slowly. At first—similar to the HEA—vacancies and small dislocation networks appear, then these start disappearing in favor of stacking-fault tetrahedra and a Frank loop.

the supplementary material). A small number of stackingfault tetrahedra appear, but seem to be unstable. Additionally, we can observe the formation of complex networks of partial dislocations. Because of the large amount of dislocation junctions, these networks must be sessile. Thus, large movements of these networks must again be a series of annihilation and creation events, and they are therefore also unstable against irradiation. In $\mathrm{Ni}$, the defects are assimilated into larger structures, such as sessile Frank dislocation loops, before they can be destroyed by the next cascade (see the video cascadeNi.avi in the supplementary material, starting at 0:40; defect close to the center of the box). As such, Ni possesses a higher volume fraction of undisturbed lattice in which the net defect creation rate is always positive. Additionally, in contrast to the HEA, the stacking-fault tetrahedra are numerous.

In the final stage, we also observe a saturation of the defect concentration in pure $\mathrm{Ni}$, while maintaining the steady state in the alloy system. The development of a steady state in the elemental structure is contrary to earlier publications, ${ }^{20}$ where a continuous growth of defect networks has been detected. The reason for a reduced growth rate of defect concentrations in $\mathrm{Ni}$ is a simulation size effect, ${ }^{47}$ since the earlier simulation used larger simulation cells compared to the current work. 
In order to examine the stability of the lattice defects in the absence of irradiation, an MD simulation at $800 \mathrm{~K}$ for $1 \mathrm{~ns}$ was performed for the initially segregated HEA. The results reveal neither large movement nor creation or dissolution of a significant number of defects, which indicates that the defects are stable and sessile even at elevated temperatures (see video reanneal-irradiated-CuNiCoFe.avi in the supplementary material).

\section{Chemical order}

In the following, we analyze whether high energy recoils are able to initiate local segregation processes, or if the SRO remains more or less constant after every subsequent cascade. Again, we use the Warren-Cowley parameters $\alpha_{1,2}^{i j}$ for the first and second neighbor shell to quantify the extent of atomic clustering. Figure 5 shows $\alpha^{\mathrm{Cu}, \mathrm{Cu}}$ for two cells: the HEA with initially random atom distribution, and the HEA after MC/MD at $400 \mathrm{~K}$ which shows segregation tendencies for $\mathrm{Cu}$. The analysis is limited to atoms in perfect FCC environments and therefore excludes atoms in defective sites introduced by the irradiation. Order parameters for pairs other than $\mathrm{Cu}-\mathrm{Cu}$ are plotted in Fig. $\mathrm{S} 1$ in the supplementary material. We find that the order parameter $\alpha_{1}^{\mathrm{Cu}, \mathrm{Cu}}$, although exhibiting initial partial segregation in the structure preordered by $\mathrm{MC} / \mathrm{MD}$, eventually converges to small negative values. The initially random structure converges to the same value. A mid-range order is always disturbed by irradiation: the parameter $\alpha_{2}^{\mathrm{Cu}, \mathrm{Cu}}$ converges to zero in both cases. This shows that the cascade has two counteracting effects: First, it locally activates the thermodynamically preferrable segregation of $\mathrm{Cu}$. Second, it concurrently randomizes the element distribution. The resulting steady state is reached after approximately 700 cascades.

Figure 6 shows the comparison of the Warren-Cowley parameters $\alpha_{1,2}$ for all pairs in the CuNiCoFe alloy for both

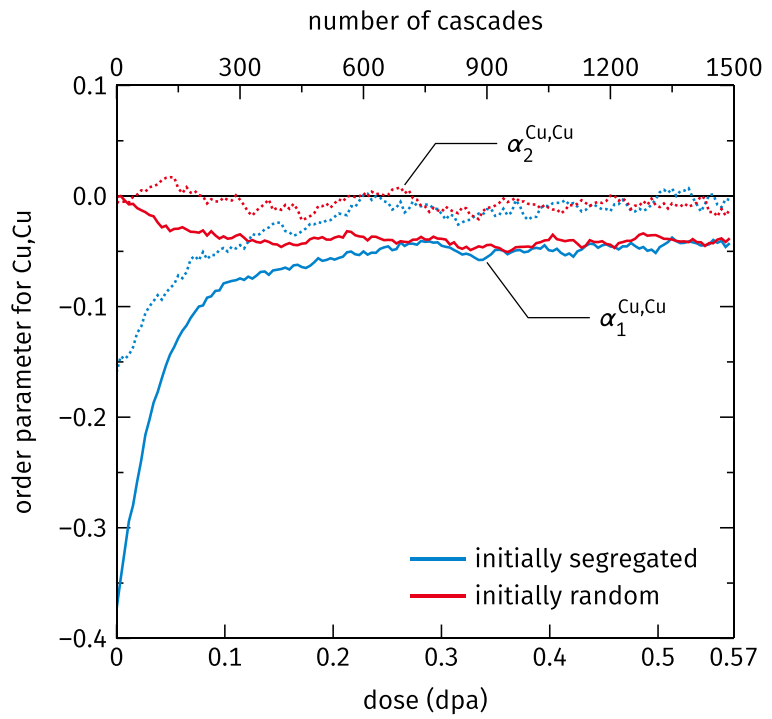

FIG. 5. Evolution of the SRO during irradiation. Only the $\mathrm{Cu}-\mathrm{Cu}$ parameters are shown, the graphs for all parameters can be found in Fig. S1 in the supplementary material. The sample which was equilibrated using VC-SGC (blue) and the one which was initially random (red) converge to the same values for the order parameters.
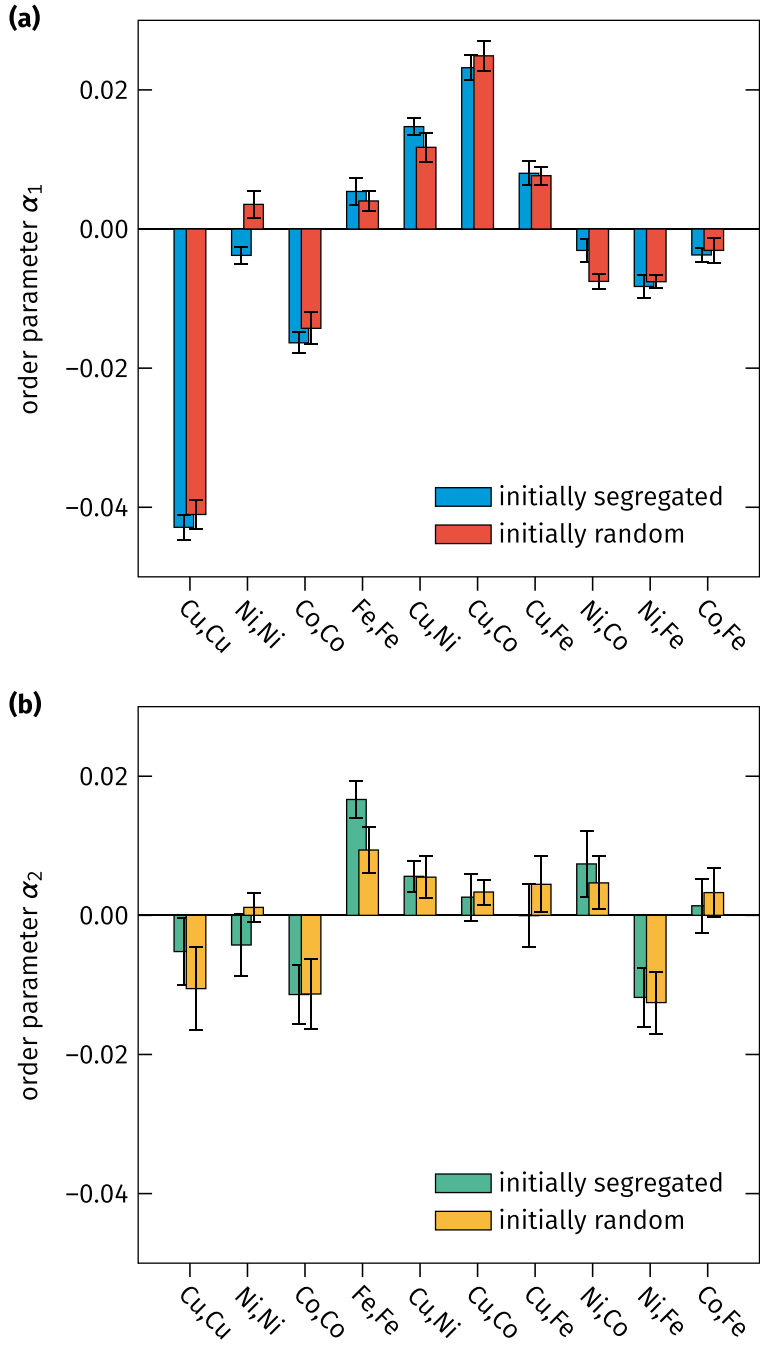

FIG. 6. Final order parameters for the first (a) and second neighbor shell (b) after irradiation with a dose of $0.57 \mathrm{dpa}$. The values are averaged over the last 10 frames of the irradiation simulation, with the error bars representing the standard deviation.

structures (pre-ordered and fully random) after the full irradiation dose. Comparing these results to those in Fig. 2 (note the scale difference in the $y$ axes), we observe that the absolute values of both parameters $\alpha_{1}$ and $\alpha_{2}$ are reduced by roughly one order of magnitude due to the irradiation. Importantly, after irradiation all pairs have practically the same $\alpha$ parameters regardless of the initial state. We also note that the $\alpha_{2}$ values are consistently smaller than the $\alpha_{1}$, indicating that the ordering effects under irradiation are limited to the nearestneighbor shell.

Based on this analysis, we conclude that while collisional cascades provide activation energy for demixing, the system is dynamically driven to a mostly random steady state, independent of the initial chemical order.

\section{POST-IRRADIATION EFFECTS}

Thermodynamic equilibration of a four-component $\mathrm{CuNiCoFe}$ alloy with a perfect FCC lattice showed a segregation tendency for $\mathrm{Cu}$ atoms. From the results of Sec. III A, we can assume that there is not only a chemical but also a sterical driving force for $\mathrm{Cu}$ to segregate from the solid 
solution into small clusters. We already concluded that the growth of $\mathrm{Cu}$ clusters is accompanied by an increasing $\mathrm{Cu}$ atomic volume. Nevertheless, thus far, we mainly concentrated on the thermodynamics of the defect-free system. Therefore, we again ran hybrid MC/MD simulations in the VC-SGC ensemble. However, this time, the irradiated sample with pre-existing defects was taken as a starting configuration. Table I lists the composition inside the lattice defects. Obviously, defect sites that offer excess volume serve as sinks for $\mathrm{Cu}$ atoms and even enhance the amount of segregated $\mathrm{Cu}$ after equilibration. In this regard, the presence of defects enhances the driving force for local decomposition. Within the defects, we can find a $\mathrm{Cu}$ proportion which is approximately $50 \%$. In contrast, during the irradiation the distribution of $\mathrm{Cu}$ atoms does not alter significantly. In Fig. 7, we visualized the atoms located within the defect structure before (left) and after (right) the equilibration. Copper atoms are displayed with a red color, while all others are shown in gray. Figures 7(c) and 7(d) highlight two defects separately. From these illustrations, we draw the conclusion that $\mathrm{Cu}$ preferentially segregates on, e.g., the edges of stacking-fault tetrahedra rather than in the stacking faults themselves. The reason lies in the higher local excess volume of these sites (cf. Fig. 3).

In conclusion, all results suggest that the mixing enthalpy of $\mathrm{Cu}$ is the decisive factor for phase stability in this case, exceeding the total entropy with decreasing temperature. The limited segregation tendencies of the perfect crystal are surpassed in the presence of defects, which act as sinks for copper.

\section{CONCLUSION}

Using a model CuNiCoFe HEA with a tendency for copper segregation, we observe that the system reaches a steady state of defect concentration and of chemical order under irradiation, irrespective of the initial structure. In contrast to pure metals, irradiation leads to less mobile point defects and therefore a larger number of isolated defects instead of recombination or agglomeration. While, e.g., $\mathrm{Ni}$ increases its defect concentration continuously with increasing irradiation dose due to the agglomeration of defects in larger, more stable structures, the HEA quickly reaches a steady state of defect creation and annihilation. Irradiation provides the thermal activation for demixing of copper, but at the same

TABLE I. Composition inside defects of the irradiated sample before and after an additional, subsequent MC/MD run. Irradiated sample averaged over 50 snapshots (500 cascades). Note that the total composition of the sample stays equimolar during MC/MD because of the variance constraint and that the composition on the intact lattice sites also stays roughly equimolar because of the comparatively low amount of defect sites.

\begin{tabular}{lcc}
\hline \hline Element & $\begin{array}{c}\text { Composition after } \\
\text { irradiation (\%) }\end{array}$ & $\begin{array}{c}\text { Composition after subsequent } \\
\text { MC/MD (\%) }\end{array}$ \\
\hline $\mathrm{Cu}$ & 26.9 & 49.4 \\
$\mathrm{Ni}$ & 24.9 & 17.1 \\
$\mathrm{Co}$ & 23.3 & 14.0 \\
$\mathrm{Fe}$ & 24.9 & 19.5 \\
\hline \hline
\end{tabular}

(a)

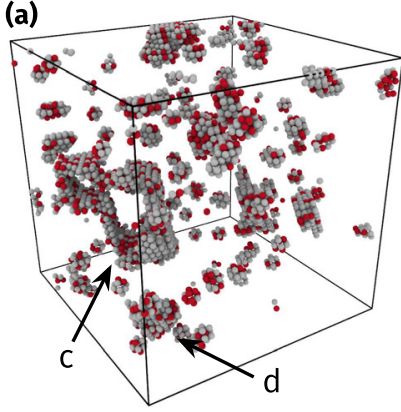

(b)

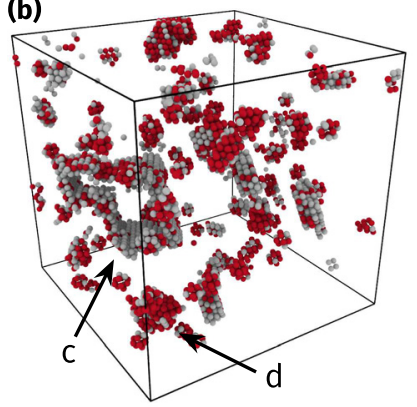

(c)
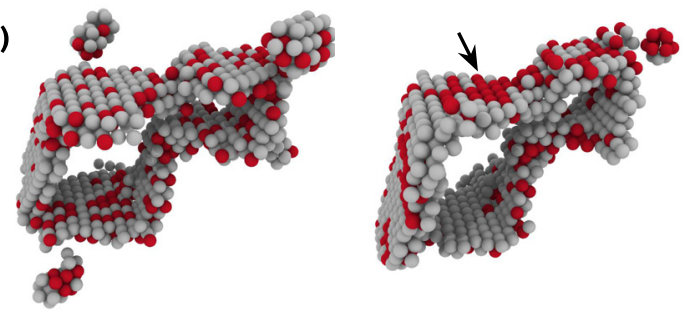

(d)

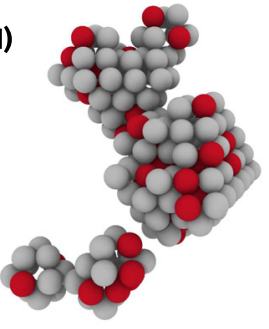

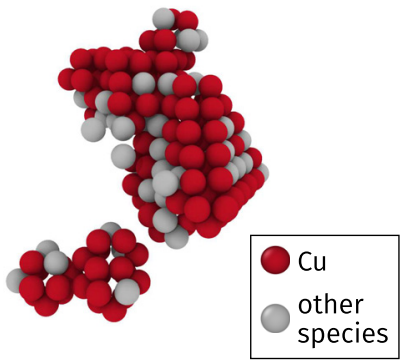

FIG. 7. VC-SGC simulations of the HEA sample after irradiation. (a) Atoms identified as defective by CNA after irradiation [same structure as the righmost snapshot in Fig. 4(a)]. Copper atoms are shown in red, all others in gray. (b) The same after subsequent simulation in the VC-SGC ensemble. The concentration of copper atoms around the defects is highly increased. Detailed views of a dislocation structure (c) and a stacking-fault tetrahedron (d) are provided.

time re-randomizes the elemental distribution. The resulting steady state of chemical order is close to a random solid solution, but still shows traces of local precipitation. Furthermore, our simulations reveal that various lattice defects act as sinks for copper, most likely since the copper atoms are the largest species and the defects provide excess volume. This clustering of copper at defects is also suppressed during irradiation for the reasons enumerated above. Together, these effects explain the high irradiation resistance of HEAs.

\section{SUPPLEMENTARY MATERIAL}

See supplementary material for videos of the irradiation simulations, a figure of the detailed evolution of SRO during irradiation, and additional data for the verification of the EAM potential.

\section{ACKNOWLEDGMENTS}

The authors gratefully acknowledge travel grants through the PPP Finland program of the Deutscher Akademischer Austauschdienst (DAAD) and K.A. acknowledges financial support by the Deutsche Forschungsgemeinschaft (DFG) 
through project Grant No. STU 611/2-1. Computing time was made available by the Technische Universität Darmstadt on the Lichtenberg cluster and the IT Center for Science, CSC, Finland.

\section{APPENDIX: VALIDATION OF THE EAM POTENTIAL}

We used an EAM potential by Zhou et al. ${ }^{24}$ for the $\mathrm{Cu}-$ Ni-Co-Fe system. This potential was tested for HEAs under driven conditions: Thin-film growth by sputtering yields comparable structures to experiment and the BCC to FCC transition is reproduced correctly. ${ }^{10,62}$ Furthermore, damage accumulation during irradiation agrees with data obtained by Rutherford backscattering spectrometry. ${ }^{35}$

We performed additional validation of the potential by comparing the properties of the elements and of binary mixtures to reference values. In a first step, we produced FCC lattices of $\mathrm{Cu}, \mathrm{Ni}, \mathrm{Co}$, and $\mathrm{Fe}$, as well as $\mathrm{HCP} \mathrm{Co}$ and $\mathrm{BCC}$ $\mathrm{Fe}$. These were minimized to obtain the $0 \mathrm{~K}$ ground state values of the lattice constants $a$ and $c$ and the cohesive energy $E_{\text {coh. }}$ The comparison to literature values in Table II reveals a good match. We then obtained the stiffness tensor using Hooke's law by calculating the stress tensors for finite deformations of the box at $1 \%$ strain. Apart from HCP cobalt, which does not occur in our samples, and FCC iron, which is too soft, the reference values are reproduced quite well. Using the procedure described in Ref. 55, we calculated the stacking fault energy $\gamma_{\mathrm{SF}}$, the unstable stacking fault energy $\gamma_{\text {USF, }}$ and the critical shear stress $\sigma_{\mathrm{SF}}$ for a stacking fault. While literature data contain large uncertainties and variations, the general trend of available data agrees with the predictions of the potential. As far as reference data are available, the same is true for the twinning faults, which we calculated using the method described in Ref. 63. The negative values for the stacking and twinning faults in Co are of course the result of the lower energy of the stable HCP structure.

Furthermore, we calculated the elastic constants of those binary alloys, for which reference data are available, i.e., in the Fe-Co-Ni system. The results are presented in Table S.1 in the supplementary material. Since the cross terms of the potential are obtained through a mixing procedure, the results match the expectations from the properties of the elements: The elastic constants match well, except for alloys containing FCC iron or HCP cobalt, which are too soft. To additionally determine the melting points of the elements and some miscible alloys, liquid-solid interface simulations were carried out. ${ }^{64}$ A simulation cell of 2000 atoms was relaxed with the correct crystal structure and lattice constant, without pressure control. An equally sized box was molten and cooled down to the desired temperature, with pressure control in the $z$ direction. The two boxes were then combined in the $z$ direction, and were let to relax with pressure control in all directions. Below the melting point, the cell will solidify and the volume of the cell will decrease, while the opposite will happen above the melting point. Due to the chosen temperature stepping, an error of around $25 \mathrm{~K}$ is expected. The results are presented in Table S.2 in the supplementary material and show that the melting points slightly deviate from the reference values, but capture the features of the phase diagrams: For the pure elements, the deviation of the melting point is below $15 \%$ of the absolute value. The melting point of HCP cobalt agrees with the literature value within the expected error of the simulation. The melting points of the different binary subsystems studied are all within $10 \%$ of the expected value. We point out that a good description of the melting points is particularly important for

TABLE II. Comparison between the potential and literature values for properties of $\mathrm{Cu}, \mathrm{Ni}, \mathrm{Co}$, and Fe. The table shows lattice constants $a$ and $c$, the cohesive energy $E_{\mathrm{coh}}$, the components of the stiffness tensor $c_{i j}$, the stacking fault energy $\gamma_{\mathrm{SF}}$, the unstable stacking fault energy $\gamma_{\mathrm{USF}}$, the critical shear stress $\sigma_{\mathrm{SF}}$ for a stacking fault, the twinning fault energy $\gamma_{\mathrm{TF}}$, the unstable twinning fault energy $\gamma_{\mathrm{UTF}}$, and the melting point $T_{\text {melt }}$.

\begin{tabular}{|c|c|c|c|c|c|c|c|c|c|c|c|c|}
\hline & \multicolumn{2}{|c|}{$\mathrm{Cu}(\mathrm{FCC})$} & \multicolumn{2}{|c|}{$\mathrm{Ni}(\mathrm{FCC})$} & \multicolumn{2}{|c|}{ Co (FCC) } & \multicolumn{2}{|c|}{$\mathrm{Co}(\mathrm{HCP})$} & \multicolumn{2}{|c|}{$\mathrm{Fe}(\mathrm{FCC})$} & \multicolumn{2}{|c|}{$\mathrm{Fe}(\mathrm{BCC})$} \\
\hline & Pot. & Ref. & Pot. & Ref. & Pot. & Ref. & Pot. & Ref. & Pot. & Ref. & Pot. & Ref. \\
\hline$a(\AA)$ & 3.615 & $3.615^{48}$ & 3.520 & $3.524^{48}$ & 3.549 & $3.545^{48}$ & 2.501 & $2.507^{48}$ & 3.628 & $3.647^{48}$ & 2.866 & $2.867^{48}$ \\
\hline$c(\AA)$ & & & & & & & 4.076 & $4.069^{48}$ & & & & \\
\hline$c / a$ & & & & & & & 1.630 & $1.623^{48}$ & & & & \\
\hline$E_{\text {coh }}(\mathrm{eV} /$ at. $)$ & -3.54 & $-3.49^{49}$ & -4.45 & $-4.44^{49}$ & -4.40 & $-4.29^{\mathrm{a} 50,51}$ & -4.41 & $-4.39^{49}$ & -4.20 & $-4.17^{52}$ & -4.29 & $-4.28^{49}$ \\
\hline$c_{11}(\mathrm{GPa})$ & 170 & $168^{48}$ & 246 & $248^{48}$ & 213 & $225^{53}$ & 263 & $307^{48}$ & 107 & $154^{54}$ & 229 & $226^{48}$ \\
\hline$c_{12}(\mathrm{GPa})$ & 122 & $122^{48}$ & 147 & $155^{48}$ & 157 & $160^{53}$ & 158 & $165^{48}$ & 98 & $122^{54}$ & 136 & $140^{48}$ \\
\hline$c_{13}(\mathrm{GPa})$ & & & & & & & 124 & $103^{48}$ & & & & \\
\hline$c_{33}(\mathrm{GPa})$ & & & & & & & 363 & $358^{48}$ & & & & \\
\hline$c_{44}(\mathrm{GPa})$ & 76 & $76^{48}$ & 125 & $124^{48}$ & 99 & $92^{53}$ & 65 & $76^{48}$ & 80 & $77^{54}$ & 117 & $116^{48}$ \\
\hline$\gamma_{\mathrm{SF}}\left(\mathrm{mJ} / \mathrm{m}^{2}\right)$ & 22 & $36-49^{55-57}$ & 97 & $133-183^{56,57}$ & -40 & & & & & & & \\
\hline$\gamma_{\mathrm{USF}}\left(\mathrm{mJ} / \mathrm{m}^{2}\right)$ & 110 & $95-210^{\text {b } 55-57}$ & 251 & $258^{56}$ & 174 & & & & & & & \\
\hline$\sigma_{\mathrm{SF}}(\mathrm{GPa})$ & 2.3 & $2.2^{55}$ & 5.1 & & 3.7 & & & & & & & \\
\hline$\gamma_{\mathrm{TF}}\left(\mathrm{mJ} / \mathrm{m}^{2}\right)$ & 23 & & 99 & & -44 & & & & & & & \\
\hline$\gamma_{\text {UTF }}\left(\mathrm{mJ} / \mathrm{m}^{2}\right)$ & 121 & $143^{56}$ & 298 & $186^{56}$ & 159 & & & & & & & \\
\hline$T_{\text {melt }}(\mathrm{K})$ & 1175 & $1357^{58,59}$ & 1513 & $1728^{58,59}$ & & & 1756 & $1768^{60}$ & & & 2044 & $1811^{61}$ \\
\hline
\end{tabular}

${ }^{\mathrm{a}}$ There are considerably different absolute values reported in the literature. We assumed a difference of $0.1 \mathrm{eV} /$ atom to the HCP structure, while the literature reports differences from $0.02 \mathrm{eV} /$ atom to $0.15 \mathrm{eV} / \mathrm{atom}$.

${ }^{\mathrm{b}}$ Value for Ref. 55 computed by numeric integration of the stress data. 
the irradiation simulations, since several previous studies show that the outcome of molecular dynamics of collision cascades depends directly on the melting point. ${ }^{65,66}$

In a next step, random solid solutions of all binary subsystems on FCC lattices were prepared. Additionally, if the binary alloy contained $\mathrm{Fe}$ or $\mathrm{Co}$, $\mathrm{BCC}$ or $\mathrm{HCP}$ lattices, respectively, were created. Different molar fractions of each alloy and lattice type were minimized to obtain the mixing enthalpy

$$
H_{\mathrm{M}}=H_{\mathrm{AB}}-\left(x_{\mathrm{A}} H_{\mathrm{A}}+x_{\mathrm{B}} H_{\mathrm{B}}\right),
$$

where $x_{\mathrm{A}}, x_{\mathrm{B}}, H_{\mathrm{A}}$, and $H_{\mathrm{B}}$ are the molar fractions and enthalpies of the constituents $\mathrm{A}$ and $\mathrm{B}$, and where $H_{\mathrm{AB}}$ is the total enthalpy of the alloy. We included mixing enthalpy curves of all binary subsystems with respect to the concentration in Figs. S2-S7 in the supplementary material and compared them to literature values. We additionally compared our results to data from the THERMO-CALC software and database. ${ }^{67,68}$ In most cases, the EAM potential correctly describes the trend observed in the literature. However, it should be noted that even different literature values do not completely agree and that there is a large spread for some systems.

In order to get a clearer picture, we investigated the miscibility gaps of the binary subsystems. For this, equimolar alloys on FCC lattices were equilibrated in the semi-grandcanonical ensemble without a variance constraint. By systematically varying $\Delta \mu$, final compositions ranging over the whole phase diagram were obtained. The simulations were performed at 300 and $800 \mathrm{~K}$. The concentration as a function of the chemical potential difference can be used to identify miscibility gaps in a binary subsystem: Given a dense mesh of $\Delta \mu_{\mathrm{A}-\mathrm{B}}$ values, miscibility gaps appear as discontinuities in the $x_{\mathrm{B}}\left(\Delta \mu_{\mathrm{A}-\mathrm{B}}\right)$ curves. Performing this analysis and comparing it to reference data showed that the potential is mostly able to reproduce phase diagram features, even though the miscibility is generally a bit too high. In the Co-containing systems, this method will not find the HCP phase, since the simulation setup was always initiated from an FCC structure. Due to the difference in the unit cell shape, the simulation suppresses the transition to HCP. We omit this investigation, since the HEA remains in the FCC structure in all conditions. The $x_{\mathrm{B}}\left(\Delta \mu_{\mathrm{A}-\mathrm{B}}\right)$ curves and a more detailed discussion of all binary subsystems can be found in Figs. S8-S13 in the supplementary material.

Finally, the lattice constants of the binary systems were calculated from these simulations by time averaging the simulation cell volume of the different binary systems at $300 \mathrm{~K}$. The data are shown in Table III and are in good agreement with experimental data. This confirms that the cross terms of the potential correctly predict the atomic volumes in alloys.

All in all, the potential performs reasonably well, reproducing the correct stable phases, densities, stiffnesses, and melting points. The limited data for stacking fault and twinning-fault energies suggest that lattice defects should be correctly reproduced. The main weakness of the potential is that the solubility of the alloys is too high in some cases, meaning that the tendencies for copper precipitation may be underestimated in the present work.
TABLE III. Lattice constants of binary random solid solutions at $300 \mathrm{~K}$. The lattice structure is FCC unless stated otherwise.

\begin{tabular}{lcc}
\hline \hline & \multicolumn{2}{c}{$a(\AA)$} \\
\cline { 2 - 3 } & Pot. & Ref. \\
\hline $\mathrm{Cu}_{3} \mathrm{Ni}_{97}$ & 3.536 & $3.526^{69,70}$ \\
$\mathrm{Cu}_{49} \mathrm{Ni}_{51}$ & 3.591 & $3.564^{69,70}$ \\
$\mathrm{Cu}_{95} \mathrm{Ni}_{5}$ & 3.643 & $3.609^{69,70}$ \\
$\mathrm{Cu}_{8} \mathrm{Co}_{92}$ & 3.568 & $3.551^{71}$ \\
$\mathrm{Cu}_{21} \mathrm{Co}_{79}$ & 3.607 & $3.577^{71}$ \\
$\mathrm{Cu}_{86} \mathrm{Co}_{14}$ & 3.636 & $3.668^{71}$ \\
$\mathrm{Cu}_{99} \mathrm{Co}_{1}$ & 3.646 & $3.674^{71}$ \\
$\mathrm{Cu}_{23} \mathrm{Fe}_{77}(\mathrm{BCC})$ & 2.890 & $2.890^{72}$ \\
$\mathrm{Cu}_{96} \mathrm{Fe}_{4}$ & 3.648 & $3.616^{72}$ \\
$\mathrm{Ni}_{26} \mathrm{Co}_{74}$ & 3.555 & $3.539^{73}$ \\
$\mathrm{Ni}_{29} \mathrm{Co}_{71}$ & 3.554 & $3.538^{73}$ \\
$\mathrm{Ni}_{49} \mathrm{Co}_{51}$ & 3.548 & $3.533^{73}$ \\
$\mathrm{Ni}_{51} \mathrm{Co}_{49}$ & 3.548 & $3.533^{73}$ \\
$\mathrm{Ni}_{72} \mathrm{Co}_{28}$ & 3.541 & $3.528^{73}$ \\
$\mathrm{Ni}_{18} \mathrm{Fe}_{82}(\mathrm{BCC})$ & 2.869 & $2.867^{61}$ \\
$\mathrm{Ni}_{39} \mathrm{Fe}_{61}$ & 3.594 & $3.595^{61}$ \\
$\mathrm{Ni}_{48} \mathrm{Fe}_{52}$ & 3.584 & $3.590^{61}$ \\
$\mathrm{Ni}_{49} \mathrm{Fe}_{51}$ & 3.582 & $3.587^{61}$ \\
$\mathrm{Ni}_{78} \mathrm{Fe}_{22}$ & 3.552 & $3.552^{61}$ \\
$\mathrm{Co}_{11} \mathrm{Fe}_{89}(\mathrm{BCC})$ & 2.869 & $2.861^{74}$ \\
$\mathrm{Co}_{24} \mathrm{Fe}_{76}$ & 2.857 & $2.860^{74}$ \\
\hline \hline & &
\end{tabular}

${ }^{1}$ Z. Wang, S. Guo, and C. T. Liu, JOM 66, 1966 (2014).

${ }^{2}$ Y. F. Ye, Q. Wang, J. Lu, C. T. Liu, and Y. Yang, Mater. Today 19, 349 (2016).

${ }^{3}$ F. Otto, Y. Yang, H. Bei, and E. P. George, Acta Mater. 61, 2628 (2013).

${ }^{4}$ E. J. Pickering and N. G. Jones, Int. Mater. Rev. 61, 183 (2016).

${ }^{5}$ C. C. Tasan, Y. Deng, K. G. Pradeep, M. J. Yao, H. Springer, and D. Raabe, JOM 66, 1993 (2014).

${ }^{6} \mathrm{~J} .-\mathrm{W}$. Yeh, JOM 65, 1759 (2013).

${ }^{7}$ M. Widom, W. P. Huhn, S. Maiti, and W. Steurer, Metall. Mater. Trans. A 45, 196 (2014).

${ }^{8}$ M.-H. Tsai and J.-W. Yeh, Mater. Res. Lett. 2, 107 (2014).

${ }^{9}$ W. H. Liu, Y. Wu, J. Y. He, Y. Zhang, C. T. Liu, and Z. P. Lu, JOM 66, 1973 (2014)

${ }^{10}$ L. Xie, P. Brault, J.-M. Bauchire, A.-L. Thomann, and L. Bedra, J. Phys. D: Appl. Phys. 47, 224004 (2014).

${ }^{11}$ F. Tian, L. K. Varga, N. Chen, L. Delczeg, and L. Vitos, Phys. Rev. B 87, 075144 (2013).

${ }^{12}$ S.-W. Kao, J.-W. Yeh, and T.-S. Chin, J. Phys: Condens. Matter 20, 145214 (2008).

${ }^{13}$ A. Haglund, M. Koehler, D. Catoor, E. P. George, and V. Keppens, Intermetallics 58, 62 (2015).

${ }^{14}$ F. Tian, L. Delczeg, N. Chen, L. K. Varga, J. Shen, and L. Vitos, Phys, Rev. B 88, 085128 (2013).

${ }^{15}$ S. Guo, Q. Hu, C. Ng, and C. T. Liu, Intermetallics 41, 96 (2013).

${ }^{16}$ C. Ng, J. Luan, Q. Wang, S. Shi, and C. Liu, J. Alloys Compd. 584, 530 (2014).

${ }^{17}$ T. Egami, W. Guo, P. D. Rack, and T. Nagase, Metall. Mater. Trans. A 45, 180 (2014).

${ }^{18}$ Y. Zhang, G. M. Stocks, K. Jin, C. Lu, H. Bei, B. C. Sales, L. Wang, L. K. Béland, R. E. Stoller, G. D. Samolyuk, M. Caro, A. Caro, and W. J. Weber, Nat. Commun. 6, 8736 (2015).

${ }^{19}$ F. Granberg, K. Nordlund, M. W. Ullah, K. Jin, C. Lu, H. Bei, L. M. Wang, F. Djurabekova, W. J. Weber, and Y. Zhang, Phys. Rev. Lett. 116, 135504 (2016).

${ }^{20}$ F. Granberg, F. Djurabekova, E. Levo, and K. Nordlund, Nucl. Inst. Methods Phys. Res., Sect. B. 393, 114 (2017).

${ }^{21}$ T. Yang, S. Xia, S. Liu, C. Wang, S. Liu, Y. Fang, Y. Zhang, J. Xue, S. Yan, and Y. Wang, Sci. Rep. 6, 32146 (2016).

${ }^{22}$ M. W. Ullah, D. S. Aidhy, Y. Zhang, and W. J. Weber, Acta Mater. 109, 17 (2016). 
${ }^{23}$ S. M. Foiles, M. I. Baskes, and M. S. Daw, Phys. Rev. B 33, 7983 (1986).

${ }^{24}$ X. W. Zhou, R. A. Johnson, and H. N. G. Wadley, Phys. Rev. B 69, 144113 (2004).

${ }^{25}$ K. T. Jacob, S. Raj, and L. Rannesh, Int. J. Mater. Res. 98, 776 (2007).

${ }^{26}$ S. Plimpton, J. Comp. Phys. 117, 1 (1995).

${ }^{27}$ B. Sadigh, P. Erhart, A. Stukowski, A. Caro, E. Martinez, and L. ZepedaRuiz, Phys. Rev. B 85, 184203 (2012).

${ }^{28}$ T. Brink, L. Koch, and K. Albe, Phys. Rev. B 94, 224203 (2016).

${ }^{29} \mathrm{~K}$. Nordlund, Parcas computer code (2016). The main principles of the molecular dynamics algorithms are presented in Refs. 45 and 75. The adaptive time step and electronic stopping algorithms are the same as in Ref. 76. The 2016 version of the code is published in the online supplementary material to Ref. 19.

${ }^{30}$ H. J. C. Berendsen, J. P. M. Postma, W. F. van Gunsteren, A. DiNola, and J. R. Haak, J. Chem. Phys. 81, 3684 (1984).

${ }^{31}$ J. F. Ziegler, J. P. Biersack, and U. Littmark, The Stopping and Range of Ions in Matter (Pergamon, New York, 1985).

${ }^{32}$ J. F. Ziegler, SRIM-96 computer code, private communication (1996).

${ }^{33}$ ASTM Standard E693-94, Standard Practice for Characterising Neutron Exposure in Iron and Low Alloy Steels in Terms of Displacements Per Atom (Dpa) (ASTM International 1994).

${ }^{34}$ K. Nordlund, S. J. Zinkle, T. Suzudo, R. S. Averback, A. Meinander, F. Granberg, L. Malerba, R. Stoller, F. Banhart, B. Weber, F. Willaime, S. Dudarev, and D. Simeone, Primary Radiation Damage in Materials: Review of Current Understanding and Proposed New Standard Displacement Damage Model to Incorporate in-Cascade Mixing and Defect Production Efficiency Effects (OECD Nuclear Energy Agency, Paris, France, 2015).

${ }^{35}$ S. Zhang, K. Nordlund, F. Djurabekova, F. Granberg, Y. Zhang, and T. S. Wang, Mater. Res. Lett. 5(6), 433-439 (2017).

${ }^{36}$ J. M. Cowley, Phys. Rev. 77, 669 (1950).

${ }^{37}$ A. Stukowski, Model. Simul. Mater. Sci. Eng. 18, 015012 (2010). http:// ovito.org/.

${ }^{38}$ G. Voronoï, J. Reine Angew. Math. 133, 97 (1908).

${ }^{39}$ G. Voronoï, J. Reine Angew. Math. 134, 198 (1908).

${ }^{40}$ G. Voronoï, J. Reine Angew. Math. 136, 67 (1909).

${ }^{41}$ W. Brostow, M. Chybicki, R. Laskowski, and J. Rybicki, Phys. Rev. B 57, 13448 (1998).

${ }^{42}$ J. D. Honeycutt and H. C. Andersen, J. Phys. Chem. 91, 4950 (1987).

${ }^{43}$ A. Stukowski, Model. Simul. Mater. Sci. Eng. 20, 045021 (2012).

${ }^{44}$ A. Stukowski, V. V. Bulatov, and A. Arsenlis, Model. Simul. Mater. Sci. Eng. 20, 085007 (2012).

${ }^{45}$ K. Nordlund, M. Ghaly, R. S. Averback, M. Caturla, T. Diaz de la Rubia, and J. Tarus, Phys. Rev. B 57, 7556 (1998).

${ }^{46}$ K. Nordlund and F. Gao, Appl. Phys. Lett. 74, 2720 (1999).

${ }^{47}$ E. Levo, F. Granberg, C. Fridlund, K. Nordlund, and F. Djurabekova, J. Nucl. Mater. 490, 323 (2017).

${ }^{48}$ CRC Handbook of Chemistry and Physics, 84th ed., edited by D. R. Lide (CRC Press, Boca Raton, Florida, USA, 2003).

${ }^{49}$ C. Kittel, Introduction to Solid State Physics, 8th ed. (John Wiley \& Sons, Inc., 2005).
${ }^{50}$ F. Cardellini and G. Mazzone, Philos. Mag. A 67, 1289 (1993).

${ }^{51}$ V. A. de la Peña O'Shea, I. de, P. R. Moreira, A. Roldán, and F. Illas, J. Chem. Phys. 133, 024701 (2010).

${ }^{52}$ M. Müller, P. Erhart, and K. Albe, J. Phys.: Condens. Matter 19, 326220 (2007).

${ }^{53}$ J. Gump, H. Xia, M. Chirita, R. Sooryakumar, M. A. Tomaz, and G. R. Harp, J. Appl. Phys. 86, 6005 (1999).

${ }^{54}$ J. Zarestky and C. Stassis, Phys. Rev. B 35, 4500 (1987).

${ }^{55}$ S. Ogata, J. Li, and S. Yip, Science 298, 807 (2002).

${ }^{56}$ Z.-H. Jin, P. Gumbsch, K. Albe, E. Ma, K. Lu, H. Gleiter, and H. Hahn, Acta Mater. 56, 1126 (2008).

${ }^{57}$ J. A. Zimmerman, H. Gao, and F. F. Abraham, Model. Simul. Mater. Sci. Eng. 8, 103 (2000).

${ }^{58}$ D. J. Chakrabarti, D. E. Laughlin, S. W. Chen, and Y. A. Chang, Phase Diagrams of Binary Nickel Alloys (ASM, Metals Park, OH, USA, 1991), Chap. $\mathrm{Cu}-\mathrm{Ni}$ (Copper-Nickel), pp. 85-95.

${ }^{59}$ D. J. Chakrabarti, D. E. Laughlin, S. W. Chen, and Y. A. Chang, Phase Diagrams of Binary Copper Alloys (ASM International, Materials Park, OH, USA, 1994), Chap. Cu-Ni (Copper-Nickel), pp. 266-270.

${ }^{60}$ T. Nishizawa and K. Ishida, Bull. Alloy Phase Diagrams 5, 161 (1984).

${ }^{61}$ L. J. Swartzendruber, V. P. Itkin, and C. B. Alcock, J. Phase Equilib. 12, 288 (1991).

${ }^{62}$ L. Xie, P. Brault, A.-L. Thomann, and J.-M. Bauchire, Appl. Surf. Sci. 285(Part B), 810 (2013).

${ }^{63}$ E. B. Tadmor and S. Hai, J. Mech. Phys. Solids 51, 765 (2003).

${ }^{64} \mathrm{~F}$. Ercolessi, O. Tomagnini, S. Iarlori, and E. Tosatti, "Molecular dynamics simulations of metal surfaces," in Nanosources and Manipulation of Atoms Under High Fields and Temperatures: Applications (Kluwer, Dordrecht, Netherlands, 1993), pp. 185-205.

${ }^{65}$ K. Nordlund, L. Wei, Y. Zhong, and R. S. Averback, Phys. Rev. B 57, R13965(R) (1998).

${ }^{66}$ K. Nordlund, K. O. E. Henriksson, and J. Keinonen, Appl. Phys. Lett. 79, 3624 (2001).

${ }^{67}$ Thermo-Calc Software, http://www.thermocalc.com for TC Binary Solutions Database, Version 1.0; accessed 26 July 2017.

${ }^{68}$ J. O. Andersson, T. Helander, L. Höglund, P. Shi, and B. Sundman, Calphad 26, 273 (2002)

${ }^{69}$ B. R. Coles, J. Inst. Met. 84, 346 (1956).

${ }^{70}$ J. K. A. Clarke and T. A. Spooner, J. Phys. D: Appl. Phys. 4, 1196 (1971).

${ }^{71}$ B. Predel, Landolt-Börnstein-Group IV Physical Chemistry Volume 5C: Ca-cd - co-zr (Springer-Verlag, Berlin/Heidelberg, 1993), Chap. Co-Cu (Cobalt-Copper).

${ }^{72}$ W. Klement, Trans Metall Soc. AIME 233, 1180 (1965).

${ }^{73}$ T. Nishizawa, S. Hao, M. Hasebe, and K. Ishida, Acta Metall. 31, 1403 (1983).

${ }^{74}$ W. Ellis and E. Greiner, Trans. ASM 29, 415 (1941).

${ }^{75}$ M. Ghaly, K. Nordlund, and R. S. Averback, Philos. Mag. A 79, 795 (1999).

${ }^{76}$ K. Nordlund, Comput. Mater. Sci. 3, 448 (1995). 